\title{
ACCURATE NumERICAL METHOD FOR Singular INITIAL-VALUE PROBlems
}

\author{
Tesfaye Aga Bullo ${ }^{1 *}$, Gemechis File Duressa ${ }^{1}$ and Gashu Gadisa Kiltu ${ }^{2}$ \\ ${ }^{1}$ Department of Mathematics, Jimma University, Jimma, Ethiopia \\ ${ }^{2}$ Department of Mathematics, Madda Walabu University, Robe, Ethiopia
}

\begin{abstract}
In this paper, an accurate numerical method is presented to find the numerical solution of the singular initial value problems. The second-order singular initial value problem under consideration is transferred into a first-order system of initial value problems, and then it can be solved by using the fifth-order Runge Kutta method. The stability and convergence analysis is studied. The effectiveness of the proposed methods is confirmed by solving three model examples, and the obtained approximate solutions are compared with the existing methods in the literature. Thus, the fifth-order Runge-Kutta method is an accurate numerical method for solving the singular initial value problems.
\end{abstract}

\section{KEYWORDS}

Singular, initial value problem, accurate solution.

\section{INTRODUCTION}

The studies of ordinary differential equations have attracted the attention of many mathematicians and physicists. A lot of problems in mathematical physics and astrophysics can be modelled by several types of differential equations. These equations have different characteristics depending on order, provided conditions, modelled problems, etc. In particular, initial value differential equations were used in the theory of stellar structure, thermal behaviour of a spherical cloud of gas, isothermal gas spheres, and thermionic currents (see [1 - 7].

Numerical solutions for the initial valued problems were presented by different researchers, for instance, Shiralashetti et. al., [8], was used the Haar wavelet collocation method; Susmita and Chakraverty [4], were used the Chebyshev Neural Network method [9] also used, an implicit numerical method for the numerical solution of singular initial value problems. Further, different applications of differential equations via numerical methods provided in [14 - 16]. Recently, Shiralashetti et. al., [8], states that the Haar wavelet collocation method to solve the linear and non-linear differential equations. Yet, there is a lack of accuracy and convergence. So that, it is important to apply and use efficient numerical methods for solving the second-order singular initial value problems.

One member of the family of Runge-Kutta methods is a fifth-order Runge Kutta method (RK5) which means, the error per step is on the order of $h^{6}$, while the total accumulated error has order $h^{5}$. Thus, in this paper, we use the fifth-order Runge Kutta method to produce a more accurate solution. 


\section{FORMUlation OF THE METHOD}

Consider the singular initial value problem of the form:

$$
y^{\prime \prime}(x)+\frac{\alpha}{x} y^{\prime}(x)+\beta f(x, y)=g(x), \quad 0<x \leq l,
$$

subject to the initial conditions

$$
y(0)=\delta, \quad y^{\prime}(0)=\eta,
$$

where $\alpha, \beta, \delta$ and $\eta$ are given constant numbers with $\alpha \neq 0$ for $y(x)$ is an unknown function. Now, dividing the interval $[0,1]$ into $N$ equal subinterval of mesh length $h$ and the mesh point is given by $x_{i}=x_{0}+i h$, for $i=1,2, \ldots, N-1$. For the sake of simplicity, denote $y\left(x_{i}\right)=y_{i}, z\left(x_{i}\right)=z_{i}, g\left(x_{i}\right)=g_{i}$, etc.

Further, let us the substitutions $z(x)=y^{\prime}(x)$ and $z^{\prime}(x)=y^{\prime \prime}(x)$, then Eq. (1) with Eq. (2) can be rewritten at the nodal point $x_{i}$ as:

$$
\begin{cases}y_{i}^{\prime}=F\left(x_{i}, y_{i}, z_{i}\right), & y(0)=\delta, \\ z_{i}^{\prime}=G\left(x_{i}, y_{i}, z_{i}\right), & z(0)=\eta,\end{cases}
$$

where $F\left(x_{i}, y_{i}, z_{i}\right)=z_{i}$ and $G\left(x_{i}, y_{i}, z_{i}\right)=g_{i}-\frac{\alpha}{x_{i}} z_{i}-\beta f\left(x_{i}, y_{i}\right)$.

To solve Eq. (3), we apply the single-step methods that require information about the solution at the point $x_{i}$ to calculate at $x_{i+1},[11,12]$. The general numerical solution of Eq. (3) using the fifth Runge Kutta method is given as:

$$
\left\{\begin{array}{l}
y_{i+1}=y_{i}+\sum_{i=1}^{5} w_{i} k_{i} \\
z_{i+1}=z_{i}+\sum_{i=1}^{5} w_{i} m_{i}
\end{array}\right.
$$

where

$$
\left\{\begin{array}{l}
k_{i}=h F\left(x_{i}+c_{i} h, y_{i}+\sum_{j=1}^{4} a_{i j} k_{j}, z_{i}+\sum_{j=1}^{4} a_{i j} m_{j}\right), \\
m_{i}=h G\left(x_{i}+c_{i} h, y_{i}+\sum_{j=1}^{4} a_{i j} k_{j}, z_{i}+\sum_{j=1}^{4} a_{i j} m_{j}\right) .
\end{array}\right.
$$


Advanced Computational Intelligence: An International Journal, Vol.8, No.1/2/3, July 2021

Thanks to Christodoulou [10], was present the fifth-order Runge Kutta method to solve a firstorder initial value problem of the form $\frac{d y}{d t}=f(t, y), y\left(t_{0}\right)=y_{0}$, which is given by the following equation:

$$
y_{n+1}=y_{n}+\frac{1}{90}\left(7 k_{1}+32 k_{3}+12 k_{4}+32 k_{5}+7 k_{6}\right) \text {, }
$$

where

$$
\begin{aligned}
k_{1} & =h f\left(x_{n}, y_{n}\right), \\
k_{2} & =f\left(t_{n}+\frac{h}{2}, y_{n}+\frac{k_{1}}{2}\right), \\
k_{3} & =f\left(t_{n}+\frac{h}{4}, y_{n}+\frac{3 k_{1}+k_{2}}{16}\right), \\
k_{4} & =f\left(t_{n}+\frac{h}{2}, y_{n}+\frac{k_{3}}{2}\right), \\
k_{5} & =f\left(t_{n}+\frac{3 h}{4}, y_{n}+\frac{1}{16}\left(-3 k_{2}+6 k_{3}+9 k_{4}\right)\right), \\
k_{6} & =f\left(t_{n}+h, y_{n}+\frac{1}{7}\left(k_{1}+4 k_{2}+6 k_{3}-12 k_{4}+8 k_{5}\right)\right) .
\end{aligned}
$$

Thus, to solve Eq. (3), the fifth-order Runge Kutta method can be re-written as:

$$
\left\{\begin{array}{l}
y_{i+1}=y_{i}+\frac{1}{90}\left(7 k_{1}+32 k_{3}+12 k_{4}+32 k_{5}+7 k_{6}\right), \\
z_{i+1}=z_{i}+\frac{1}{90}\left(7 m_{1}+32 m_{3}+12 m_{4}+32 m_{5}+7 m_{6}\right) .
\end{array}\right.
$$

where: $k_{1}=F\left(x_{i}, y_{i}, z_{i}\right)$,

$$
\begin{gathered}
m_{1}=G\left(x_{i}, y_{i}, z_{i}\right), \\
k_{2}=h F\left(x_{i}+\frac{h}{2}, y_{i}+\frac{k_{1}}{2}, z_{i}+\frac{m_{1}}{2}\right), \\
m_{2}=h G\left(x_{i}+\frac{h}{2}, y_{i}+\frac{k_{1}}{2}, z_{i}+\frac{m_{1}}{2}\right), \\
k_{3}=h F\left(x_{i}+\frac{h}{4}, y_{i}+\frac{1}{16}\left(3 k_{1}+k_{2}\right), z_{i}+\frac{1}{16}\left(3 m_{1}+m_{2}\right)\right), \\
m_{3}=h G\left(x_{i}+\frac{h}{4}, y_{i}+\frac{1}{16}\left(3 k_{1}+k_{2}\right), z_{i}+\frac{1}{16}\left(3 m_{1}+m_{2}\right)\right), \\
k_{4}=h F\left(x_{i}+\frac{h}{2}, y_{i}+\frac{k_{3}}{2}, z_{i}+\frac{m_{3}}{2}\right), \\
k_{5}=h F\left(x_{i}+\frac{3 h}{4}, y_{i}+\frac{1}{16}\left(-3 k_{2}+6 k_{3}+9 k_{4}\right), z_{i}+\frac{1}{16}\left(-3 m_{2}+6 m_{3}+9 m_{4}\right)\right), \\
m_{5}=h G\left(x_{i}+\frac{3 h}{4}, y_{i}+\frac{1}{16}\left(-3 k_{2}+6 k_{3}+9 k_{4}\right), z_{i}+\frac{1}{16}\left(-3 m_{2}+6 m_{3}+9 m_{4}\right)\right),
\end{gathered}
$$


Advanced Computational Intelligence: An International Journal, Vol.8, No.1/2/3, July 2021

$$
\begin{aligned}
& k_{6}=h F\left(x_{i}+h, y_{i}+\frac{1}{7}\left(k_{1}+4 k_{2}+6 k_{3}-12 k_{4}+8 k_{5}\right), z_{i}+\frac{1}{7}\left(m_{1}+4 m_{2}+6 m_{3}-12 m_{4}+8 m_{5}\right)\right), \\
& m_{6}=h G\left(x_{i}+h, y_{i}+\frac{1}{7}\left(k_{1}+4 k_{2}+6 k_{3}-12 k_{4}+8 k_{5}\right), z_{i}+\frac{1}{7}\left(m_{1}+4 m_{2}+6 m_{3}-12 m_{4}+8 m_{5}\right)\right) .
\end{aligned}
$$

In the determination of the parameters, since the terms are up to $O\left(h^{5}\right)$ be compared, the truncation error is $O\left(h^{6}\right)$ and the order of method is $O\left(h^{5}\right)$ [11 - 13].

The method is given in Eq. (6) is applied on Eq. (3) for $i \neq 0$, (i.e $x_{i} \neq x_{0}=0$ ), So that, it is necessary to modify the given second-order singular initial value problems at $i=0$, as follow:

\section{Modification at Singular}

The fifth-order Runge Kutta method given in Eq. (6) cannot be used for the stated problem at $i=0$, since it is not defined at $x=x_{0}$. Hence we have a modified form on Eq. (1) at the singular point $x=x_{0}=0$ as:

$$
y^{\prime \prime}\left(x_{0}\right)+\lim _{x \rightarrow 0} \frac{\alpha}{x} y^{\prime}(x)+\beta f\left(x_{0}, y_{0}\right)=g\left(x_{0}\right) .
$$

Using L. Hospital's Rule of limit evaluation, we obtain:

$$
\begin{aligned}
& (1+\alpha) y^{\prime \prime}\left(x_{0}\right)+\beta f\left(x_{0}, y_{0}\right)=g\left(x_{0}\right) \text { at } x=x_{0}=0, \\
& y^{\prime \prime}\left(x_{0}\right)=\frac{1}{1+\alpha}\left(g\left(x_{0}\right)-\beta f\left(x_{0}, y_{0}\right)\right), \text { for } \alpha \neq-1 .
\end{aligned}
$$

Thus, from the relations $y_{i}^{\prime}=z_{i}$, the above equation re-written as:

$$
z_{0}^{\prime}=\frac{1}{1+\alpha}\left(g_{0}-\beta f\left(x_{0}, y_{0}\right)\right), \quad z(0)=\eta
$$

Now, by applying Eq. (6) on Eq. (3) for $i \neq 0$ and on Eq. (7) for $i=0$, we can find stable and more accurate approximate solutions for the proposed problem.

\section{STABility ANALYSIS}

The problem in Eq. (1) is reduced into Eq. (3) and let take the second equation from Eq. (3), then we have:

$$
z_{i}^{\prime}=G\left(x_{i}, y_{i}, z_{i}\right), \quad z(0)=\eta,
$$

where $G\left(x_{i}, y_{i}, z_{i}\right)=g_{i}-\frac{\alpha}{x_{i}} z_{i}-\beta f\left(x_{i}, y_{i}\right)$. 
The nonlinear function Eq. (8) can be linearized by expanding the function $G$ in Taylor series about the point $\left(x_{0}, y_{0}, z_{0}\right)$ and truncating it after the first term as:

$$
\begin{aligned}
z^{\prime}= & G\left(x_{0}, y_{0}, z_{0}\right)+\left(x-x_{0}\right) \frac{\partial G}{\partial x}\left(x_{0}, y_{0}, z_{0}\right)+ \\
& \left(y-y_{0}\right) \frac{\partial G}{\partial y}\left(x_{0}, y_{0}, z_{0}\right)+\left(z-z_{0}\right) \frac{\partial G}{\partial z}\left(x_{0}, y_{0}, z_{0}\right) .
\end{aligned}
$$

By the differentiation rules of the function of several variables Eq. (9) can be written as:

$$
\begin{aligned}
z^{\prime}= & g_{0}-\frac{\alpha}{x_{0}} z_{0}-\beta f\left(x_{0}, y_{0}\right)+\left(x-x_{0}\right)\left(g_{0}^{\prime}+\frac{\alpha}{x_{0}^{2}} z-\frac{\alpha}{x_{0}} z_{0}^{\prime}-\beta \frac{\partial f}{\partial x}\left(x_{0}, y_{0}\right)\right) \\
& -\beta\left(y-y_{0}\right) \frac{\partial f}{\partial y}\left(x_{0}, y_{0}\right)+\left(z-z_{0}\right)\left(-\frac{\alpha}{x_{0}} z_{0}^{\prime}\right) .
\end{aligned}
$$

Using Eq. (12) and substitution, we get:

$$
\begin{aligned}
z^{\prime}= & g_{0}-\alpha\left(\frac{1}{1+\alpha}\left(g_{0}-\beta f\left(x_{0}, y_{0}\right)\right)\right)-\beta f\left(x_{0}, y_{0}\right)+\left(x-x_{0}\right)\left(g_{0}^{\prime}+\frac{\alpha}{2}\left(\frac{1}{1+\alpha}\left(g_{0}^{\prime}-\beta \frac{\partial f}{\partial x}\left(x_{0}, y_{0}\right)\right)\right)\right. \\
& \left.-\alpha\left(\frac{1}{1+\alpha}\left(g_{0}^{\prime}-\beta \frac{\partial f}{\partial x}\left(x_{0}, y_{0}\right)\right)\right)-\beta \frac{\partial f}{\partial x}\left(x_{0}, y_{0}\right)\right)-\beta\left(y-y_{0}\right) \frac{\partial f}{\partial y}\left(x_{0}, y_{0}\right) \\
& +\left(z-z_{0}\right)\left(-\alpha \frac{1}{1+\alpha}\left(g_{0}^{\prime}-\beta \frac{\partial f}{\partial x}\left(x_{0}, y_{0}\right)\right)\right) .
\end{aligned}
$$

This can be re-written in the form of:

$$
z^{\prime}=\lambda z+c
$$

where $\lambda=-\alpha\left(\frac{1}{1+\alpha}\left(g_{0}^{\prime}-\beta \frac{\partial f}{\partial x}\left(x_{0}, y_{0}\right)\right)\right.$,

$$
\begin{aligned}
c= & g_{0}-\alpha z_{0}^{\prime}-\beta f\left(x_{0}, y_{0}\right)+\left(x-x_{0}\right)\left(g_{0}^{\prime}-\alpha\left(\frac { 1 } { 1 + \alpha } \left(g_{0}^{\prime}-\right.\right.\right. \\
& \left.\left.\beta \frac{\partial f}{\partial x}\left(x_{0}, y_{0}\right)\right)-\beta \frac{\partial f}{\partial x}\left(x_{0}, y_{0}\right)\right)-\beta\left(y-y_{0}\right) \frac{\partial f}{\partial y}\left(x_{0}, y_{0}\right)+\alpha\left(\frac{1}{1+\alpha}\left(g_{0}^{\prime}-\beta \frac{\partial f}{\partial x}\left(x_{0}, y_{0}\right)\right) z_{0},\right.
\end{aligned}
$$

Which is linear in the variable $z$, since dividing both sides of Eq. (10) by $\lambda$, we obtain

$$
\begin{aligned}
& \frac{z^{\prime}}{\lambda}=z+\frac{c}{\lambda} \text { and let } u=z+\frac{c}{\lambda}, \text { then we have: } \\
& z=u-\frac{c}{\lambda} .
\end{aligned}
$$

Substituting Eq. (11) into Eq. (10), we get the following: $\left(u-\frac{c}{\lambda}\right)^{\prime}=\lambda\left(u-\frac{c}{\lambda}\right)+c$

$$
u^{\prime}=\lambda u
$$


which is called the linear test equation for the non-linear Eq. (8). The solution of the test equation, Eq. (8) is:

$$
u=u_{0}\left(e^{\lambda h}\right)^{n}
$$

where $u_{0}$ is constant.

Now, by applying Eq. (5) on Eq. (12), we have:

$$
\begin{aligned}
& k_{1}=\lambda h u_{i}, \\
& k_{2}=\lambda h u_{i}\left(1+\frac{1}{2} \lambda h\right), \\
& k_{3}=h \lambda u_{i}+\frac{h^{2} \lambda^{2}}{4} u_{i}+\frac{h^{3} \lambda^{3}}{32} u_{i}, \\
& k_{4}=\lambda h u_{i}\left(1+\frac{\lambda h}{2}+\frac{h^{2} \lambda^{2}}{8}+\frac{h^{3} \lambda^{3}}{64}\right), \\
& k_{5}=\lambda h u_{i}+\frac{3}{4} \lambda^{2} h^{2} u_{i}+\frac{9}{32} \lambda^{3} h^{3} u_{i}+\frac{21}{256} h^{4} \lambda^{4} u_{i}+\frac{9}{1024} \lambda^{5} h^{5} u_{i}, \\
& k_{6}=\lambda h u_{i}+\lambda^{2} h^{2} u_{i}+\frac{1}{2} \lambda^{3} h^{3} u_{i}+\frac{15}{112} h^{4} \lambda^{4} u_{i}+\frac{15}{224} h^{5} \lambda^{5} u_{i}+\frac{9}{896} \lambda^{6} h^{6} u_{i}
\end{aligned}
$$

By substituting the values of $k_{1}$ and $k_{3}-k_{6}$ into

$$
u_{i+1}=u_{i}+\frac{1}{90}\left(7 k_{1}+32 k_{3}+12 k_{4}+32 k_{5}+7 k_{6}\right) \text {, }
$$

We obtain:

$$
u_{i+1}=E(\lambda h) u_{i}
$$

where: $E(\lambda h)=1+\lambda h+\frac{1}{2} \lambda^{2} h^{2}+\frac{1}{6} \lambda^{3} h^{3}+\frac{1}{24} \lambda^{4} h^{4}+\frac{1}{120} \lambda^{5} h^{5}+\frac{1}{1280} \lambda^{6} h^{6}$.

The errors in numerical computation don't grow, if the propagation error tends to zero or if at least bounded, [13]. Now, from Eq. (13), it is easily observed, the exact value of $u\left(x_{i}\right)$ increases for the constant $\lambda>0$ and decreases for $\lambda<0$ with the factor of $e^{\lambda h}$. While from Eq. (14) the approximate value of $u_{i}$ increases or decreases with the factor of $E(\lambda h)$. If $\lambda h>0$, then $e^{\lambda h} \geq 1$; So the fifth-order Runge Kutta method is relatively stable. If $\lambda h<0$, (i.e., $\lambda<0$ ) then the fifth-order Runge Kutta method is stable in the interval of $-5.604<\lambda h<0$.

\section{NUMERICAL EXAMPLES AND RESUlTS}

To demonstrate the applicability of the methods, three model singular initial value problems have been considered. These examples have been chosen because they have been widely discussed in the literature and their solutions are available for comparison. 
Advanced Computational Intelligence: An International Journal, Vol.8, No.1/2/3, July 2021

Example 1: Consider the singular initial value problem:

$$
y^{\prime \prime}+\frac{2}{x} y^{\prime}-2\left(2 x^{2}+3\right) y=0, \quad 0<x \leq 1,
$$

subject to the initial conditions: $y(0)=1, y^{\prime}(0)=0$ and its exact solution is given as $y(x)=e^{x^{2}}$

Table 1: The comparison of pointwise relative errors at $h=0.1$ for Example 1.

\begin{tabular}{|c|c|c|c|}
\hline & Exact Solution & \multicolumn{2}{|c|}{ Pointwise relative errors } \\
\hline$x$ & & Method [4] & $\begin{array}{c}\text { Our method } \\
\text { (RK5) }\end{array}$ \\
\hline 0.1 & 1.010050167084168 & 0.0007 & $2.2109 \mathrm{e}-05$ \\
\hline 0.2 & 1.040810774192388 & 0.0013 & $6.6993 \mathrm{e}-05$ \\
\hline 0.3 & 1.094174283705210 & 0.0003 & $7.9959 \mathrm{e}-05$ \\
\hline 0.4 & 1.173510870991810 & 0.0134 & $8.5615 \mathrm{e}-05$ \\
\hline 0.5 & 1.284025416687741 & 0.0026 & $8.8478 \mathrm{e}-05$ \\
\hline 0.6 & 1.433329414560340 & 0.0021 & $9.0031 \mathrm{e}-05$ \\
\hline 0.7 & 1.632316219955379 & 0.0085 & $9.0904 \mathrm{e}-05$ \\
\hline 0.8 & 1.896480879304951 & 0.0041 & $9.1408 \mathrm{e}-05$ \\
\hline 0.9 & 2.247907986676471 & 0.0087 & $9.1714 \mathrm{e}-05$ \\
\hline 1.0 & 2.718281828459046 & 0 & $9.1923 \mathrm{e}-05$ \\
\hline
\end{tabular}

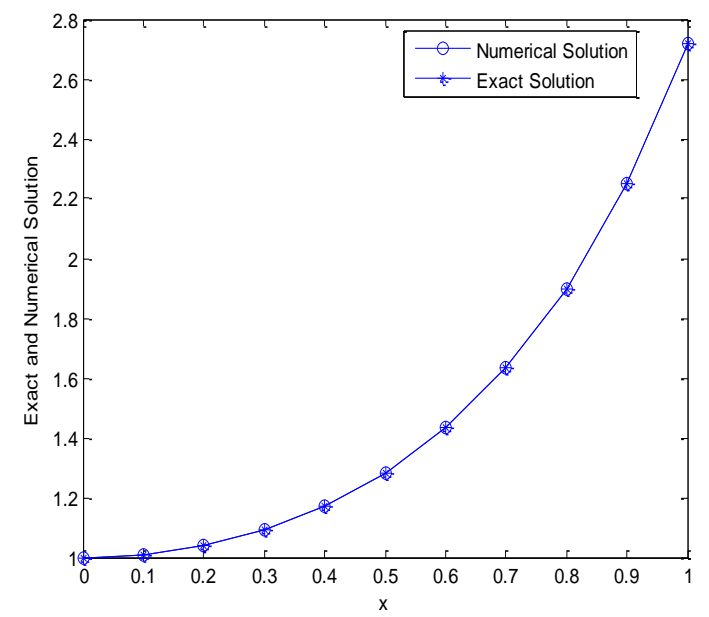

Figure 1: The Relationship between the Exact and Numerical solutions for Example 1.

Example 2: Consider the non-linear singular initial value problem:

$$
y^{\prime \prime}+\frac{2}{x} y^{\prime}+4\left(2 e^{y}+e^{\frac{y}{2}}\right)=0, \quad 0<x \leq 1,
$$

with the initial conditions $y(0)=0, y^{\prime}(0)=0$. Its exact solution is $y(x)=-2 \log \left(1+x^{2}\right)$. 
Advanced Computational Intelligence: An International Journal, Vol.8, No.1/2/3, July 2021

Table 2: The comparison of Maximum Absolute errors for Example 2.

\begin{tabular}{|c|c|c|}
\hline$N$ & Method [8] & Our method (RK5) \\
\hline 8 & $4.5110 \mathrm{e}-04$ & $3.5374 \mathrm{e}-04$ \\
\hline 16 & $1.0863 \mathrm{e}-04$ & $2.5646 \mathrm{e}-05$ \\
\hline 32 & $3.0001 \mathrm{e}-05$ & $1.7753 \mathrm{e}-06$ \\
\hline 64 & $7.1538 \mathrm{e}-06$ & $1.1832 \mathrm{e}-07$ \\
\hline 128 & $1.2981 \mathrm{e}-06$ & $7.7075 \mathrm{e}-09$ \\
\hline 256 & $4.3423 \mathrm{e}-07$ & $4.9457 \mathrm{e}-10$ \\
\hline
\end{tabular}

Table 3: Pointwise Absolute errors for Example 2 at different values of mesh length $h$.

\begin{tabular}{|c|c|c|c|c|}
\hline$x$ & $h=0.1$ & $h=0.02$ & $h=0.01$ & $h=0.001$ \\
\hline 0.1 & $4.4016 \mathrm{e}-05$ & $2.9774 \mathrm{e}-07$ & $2.0257 \mathrm{e}-08$ & $2.1701 \mathrm{e}-12$ \\
\hline 0.2 & $1.3151 \mathrm{e}-04$ & $3.1078 \mathrm{e}-07$ & $2.0126 \mathrm{e}-08$ & $2.0735 \mathrm{e}-12$ \\
\hline 0.3 & $1.5161 \mathrm{e}-04$ & $2.9602 \mathrm{e}-07$ & $1.8848 \mathrm{e}-08$ & $1.9144 \mathrm{e}-12$ \\
\hline 0.4 & $1.5196 \mathrm{e}-04$ & $2.6984 \mathrm{e}-07$ & $1.7012 \mathrm{e}-08$ & $1.7132 \mathrm{e}-12$ \\
\hline 0.5 & $1.4290 \mathrm{e}-04$ & $2.3772 \mathrm{e}-07$ & $1.4876 \mathrm{e}-08$ & $1.4879 \mathrm{e}-12$ \\
\hline 0.6 & $1.2890 \mathrm{e}-04$ & $2.0307 \mathrm{e}-07$ & $1.2625 \mathrm{e}-08$ & $1.2544 \mathrm{e}-12$ \\
\hline 0.7 & $1.1251 \mathrm{e}-04$ & $1.6827 \mathrm{e}-07$ & $1.0391 \mathrm{e}-08$ & $1.0258 \mathrm{e}-12$ \\
\hline 0.8 & $9.5351 \mathrm{e}-05$ & $1.3488 \mathrm{e}-07$ & $8.2663 \mathrm{e}-09$ & $8.0946 \mathrm{e}-13$ \\
\hline 0.9 & $7.8442 \mathrm{e}-05$ & $1.0388 \mathrm{e}-07$ & $6.3049 \mathrm{e}-09$ & $6.1151 \mathrm{e}-13$ \\
\hline 1.0 & $6.2402 \mathrm{e}-05$ & $7.5743 \mathrm{e}-08$ & $4.5336 \mathrm{e}-09$ & $4.3521 \mathrm{e}-13$ \\
\hline
\end{tabular}

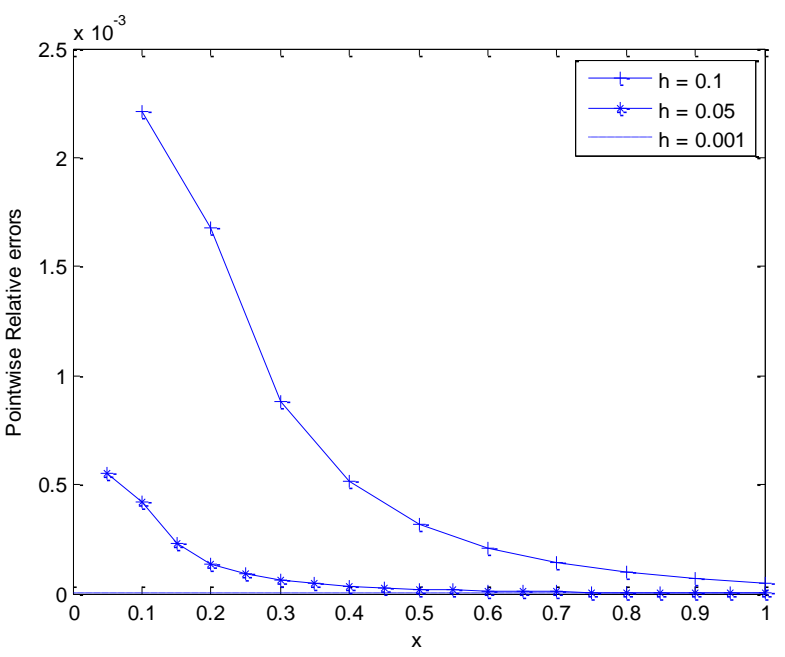

Figure 2: Relative pointwise errors decrease as the mesh size decreases for Example 2.

Example 3: Consider the following Lane-Emden equation:

$$
y^{\prime \prime}+\frac{2}{x} y^{\prime}+y^{5}=0, \quad 0 \leq x \leq 1,
$$

With the initial conditions $y(0)=1, y^{\prime}(0)=0$; whose exact solution is: $y(x)=\frac{1}{\sqrt{\left(1+\frac{x^{2}}{3}\right)}}$. 
Advanced Computational Intelligence: An International Journal, Vol.8, No.1/2/3, July 2021

Table 4: The comparison of pointwise relative errors at $h=0.1$ for Example 3.

\begin{tabular}{|c|c|c|c|}
\hline \multirow{2}{*}{$x$} & \multirow{2}{*}{ Exact solution } & \multicolumn{2}{|c|}{ Pointwise Relative errors } \\
\cline { 3 - 4 } & & Method [4] & Our method (RK5) \\
\hline 0.1 & $9.9834 \mathrm{e}-01$ & 0.0002 & $1.8472 \mathrm{e}-06$ \\
\hline 0.2 & $9.9340 \mathrm{e}-01$ & 0.0001 & $5.6146 \mathrm{e}-06$ \\
\hline 0.3 & $9.8533 \mathrm{e}-01$ & 0.0046 & $6.6915 \mathrm{e}-06$ \\
\hline 0.4 & $9.7435 \mathrm{e}-01$ & 0.0032 & $7.0461 \mathrm{e}-06$ \\
\hline 0.5 & $9.6077 \mathrm{e}-01$ & 0.0076 & $7.0582 \mathrm{e}-06$ \\
\hline 0.6 & $9.4491 \mathrm{e}-01$ & 0.0038 & $6.8632 \mathrm{e}-06$ \\
\hline 0.7 & $9.2715 \mathrm{e}-01$ & 0.0032 & $6.5281 \mathrm{e}-06$ \\
\hline 0.8 & $9.0784 \mathrm{e}-01$ & 0.0002 & $6.0945 \mathrm{e}-06$ \\
\hline 0.9 & $8.8736 \mathrm{e}-01$ & 0.0044 & $5.5917 \mathrm{e}-06$ \\
\hline 1.0 & $8.6603 \mathrm{e}-01$ & 0.0009 & $5.0421 \mathrm{e}-06$ \\
\hline
\end{tabular}

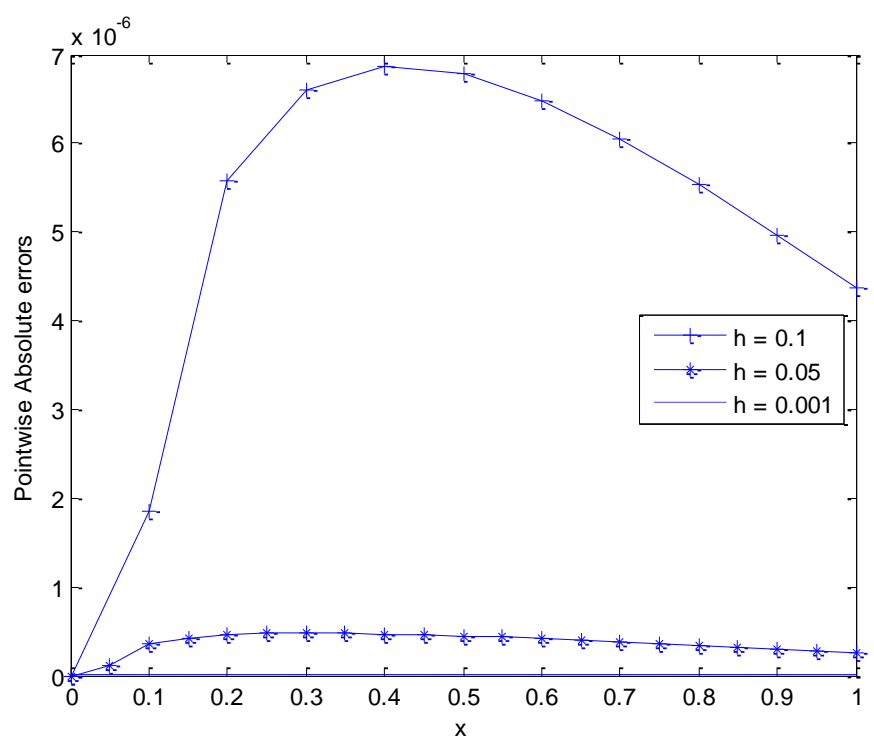

Figure 3: Absolute errors decrease as the number of mesh size $h$ decreases for Example 3.

\section{DisCUSSION AND CONCLUSION}

In this paper, the fifth-order Runge Kutta method has been presented for solving the second-order singular initial value problems. First, after the given interval or domain is discretized, the problem is reduced into a system of two first-order initial value problems, and then the obtained system is solved by using the fifth-order Runge Kutta method. Its stability analysis has been established and the method is stable in the interval of $-5.604<\lambda h<0$. To validate the applicability of the proposed method, model examples have been considered and solved for different values of mesh sizes. As it can be observed from the numerical results presented in Tables 1 - 4 and graphs (Figure 1), the present method approximates the exact solution very well and compared with the numerical results presented by Susmita and Chakraverty [4] and Shiralashetti et al., [8]. Furthermore, it observed from Tables 1, 2, and 4, the approximate solution presented in this paper is more accurate than the approximate solutions presented by the mentioned kinds of literature. Generally, all the pointwise absolute errors, pointwise relative errors, and maximum absolute errors decrease rapidly as the number of mesh size $h$ decreases, and the graphs (Figs. 2 and 3) reflect that the pointwise relative and absolute errors decrease 
Advanced Computational Intelligence: An International Journal, Vol.8, No.1/2/3, July 2021

rapidly as the number of mesh size decreases. Thus, the fifth-order Runge Kutta method is a stable, more accurate, and convergent method to find the approximate solution of the secondorder singular initial value problems.

\section{REFERENCES}

[1] Batiha, B. (2009). Numerical solution of a class of singular second-order IVPs by variational iteration method. International Journal of Mathematical Analysis, 3(40), 1953-1968.

[2] Hasan, M. K., Ahamed, M. S., Huq, M. A., Alam, M. S., \& Hossain, M. B. (2014). An implicit method for the numerical solution of second-order singular initial value problems. The Open Mathematics Journal, 7(1).

[3] Turkyilmazoglu, M. (2013). Effective computation of exact and analytic approximate solutions to singular nonlinear equations of Lane-Emden-Fowler type. Applied Mathematical Modelling, 37(1415), 7539-7548.

[4] Mall, S., \& Chakraverty, S. (2015). Numerical solution of nonlinear singular initial value problems of Emden-Fowler type using Chebyshev Neural Network method. Neurocomputing, 149, 975-982.

[5] Kiltu, G. G., \& Duressa, G. F. (2019). Accurate numerical method for Liénard nonlinear differential equations. Journal of Taibah University for Science, 13(1), 740-745.

[6] Kiltu, G. G., Duressa, G. F., \& Bullo, T. A. (2020). Numerical treatment of singularly perturbed delay reaction-diffusion equations. International Journal of Engineering, Science and Technology, 12(1), $15-24$.

[7] Siraj, M. K., Duressa, G. F., \& Bullo, T. A. (2019). Fourth-order stable central difference with Richardson extrapolation method for second-order self-adjoint singularly perturbed boundary value problems. Journal of the Egyptian Mathematical Society, 27(1), 1-14.

[8] Shiralashetti, S. C., Deshi, A. B., \& Desai, P. M. (2016). Haar wavelet collocation method for the numerical solution of singular initial value problems. Ain Shams Engineering Journal, 7(2), 663-670.

[9] Mall, S., \& Chakraverty, S. (2014). Chebyshev neural network-based model for solving Lane-Emden type equations. Applied Mathematics and Computation, 247, 100-114.

[10] Christodoulou, N. S. (2009). An algorithm using Runge-Kutta methods of orders 4 and 5 for systems of ODEs. International journal of numerical methods and applications, 2(1), 47-57.

[11] File, G., \& Aga, T. (2016). Numerical solution of quadratic Riccati differential equations. Egyptian Journal of basic and applied sciences, 3(4), 392-397.

[12] Grewal B'S, (202) Numerical method in engineering and science with programs in FORTRAN 77, C and $\mathrm{c}++$, Khanna publisher sixth edition.

[13] Jain, M. K., Iyengar, S. R. K., \& Jain, R. K. (2007). Numerical methods for scientific and Engineering Computations; equations.

[14] Bouhous, A. (2015). Artificial Neural Network In The Design Of Rectangular Microstrip Antenna. Advanced Computational Intelligence: An International Journal (ACII), 2(2).

[15] Jensi, R., \& Jiji, G. W. (2015). Hybrid data clustering approach using k-means and flower pollination algorithm. arXiv preprint arXiv:1505.03236.

[16] Kusi, G. R., Bullo, T. A., \& Duressa, G. F. (2021). Quartic Non-polynomial Spline Method for Singularly Perturbed Differential-difference Equation with Two Parameters. Journal of Engineering Advancements, 9-15. 


\section{AUTHORS}

TESFAYA AGA BULLO received B.Ec. from Addis Ababa University, and M.Sc. from Bahr Dar University. Currently, he is a Ph.D. scholar in Jimma University. His research interests are computational mathematics, numerical solution of singularly perturbed boundary value problems. He has published more than 20 research articles in reputable journals.

GEMECHIS FILE DURESSA received his M.Sc. degree from Addis Ababa University, Ethiopia and Ph.D. degree from National Institute of Technology, Warangal, India. He is currently working as an Associate professor of Mathematics at Jimma University. His research interests include Numerical Methods for Singularly Perturbed Differential Equations (both ODE and PDE). As far as this, he has published more than 70 research articles in reputable journals.

GASHU GADISA KILTU received B.Sc and M.Sc. from Jimma University, and currently he is an Assistant Professor in Mathematics at Madda Walabu University. His research interests are computational mathematics, numerical solution of singularly perturbed problems. He has published more than 15 research articles in reputable journals.
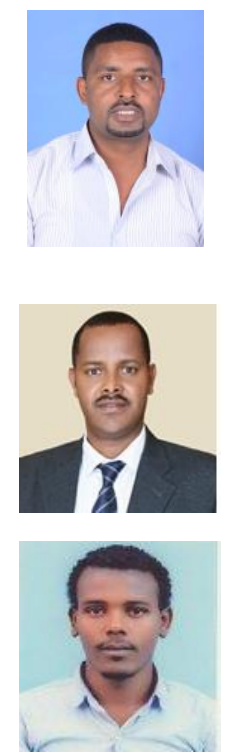